\title{
Studies suggest alternatives to amalgam as a retrograde filling material for apicectomy
}

\author{
In patients who require apicectomy and retrograde obturation, which filling \\ material is most effective?
}

\begin{abstract}
Niederman R, Theodosopoulou NJ. A systematic review of in vivo retrograde obturation materials. Int Endod J 2003; 36:577-585
\end{abstract}

Data sources Sources were Medline and the Cochrane Library.

Study selection Studies included were in vivo with human subjects, had experimental and control groups, and gave quantitative results in English, German or French.

Data extraction and synthesis Success and failure rates were derived from randomised controlled trials (RCT), clinical controlled trials (CCT), cohort studies (CS) and case-controlled studies (CCS). Qualitative synthesis of results was performed.

Results Two RCT, six CCT and 14 CCS were identified. The two RCT suggest that glass ionomer may be more effective than amalgam, conversley one CCT showed amalgam to be more effective. CCTs also suggest that EBA (reinforced zinc oxide eugenol) cement, composite with GLUMA (Bayer AG., Leverkusen, Germany) and gold leaf retrograde filling may be more effective than amalgam. A further CCT suggested that gutta-percha used as a retrograde filing is less effective than when used following an orthograde approach.

Conclusions Based on the outcome of two RCT, glass ionomer appears as effective as amalgam. EBA cement, composite with GLUMA and gold leaf and orthograde gutta-percha may also be as effective as amalgam. Evidence is limited, however, and further research is needed.

\section{Commentary}

Retrograde root canal filling after apicectomy is regarded as an important procedure to secure the seal of the apical end of root canals inaccessible to orthograde endodontic filling. For many years, amalgam has been accepted as the material of choice for retrofilling in endodontic surgery. Recently, many researchers have questioned the suitability of amalgam as a retroseal because concern over free mercury and poor results in leakage studies carried out in vitro.

The importance of this review article was its comparison of the clinical outcome of the use of different retroseal materials, not just within laboratory studies.

Many researchers look for alternative materials for retrofilling, such as glass ionomer cement, gold foil, EBA cement or cyanoacrylate. Many of these studies for retrofilling material were carried out only in vitro, as illustrated here in the result of the review authors' Medline search. Of 324 papers initially identified, 108 papers reported in vitro studies, whereas in vivo studies were limited to only 32. This made it difficult to evaluate the clinical significance of new retrofilling materials in the clinical setting.

Both the efficacy of newly introduced retrofilling materials and the success rate of amalgam retrofilling as a control therapy was distributed very broadly, at $51.9-95.7 \%$ in the extracted papers. This heterogeneity made it difficult to combine the results in a metaanalysis and to interpret the result clearly. The wide deviation of success rates might be due to the lack of standardisation of surgical procedures and inclusion/exclusion criteria of cases.

The number-needed-to-treat (NNT) was mainly used in this review to compare the clinical significance of each material. This is a useful index with which to demonstrate the number of teeth that need to be filled with experimental material to prevent one additional failure. When I recalculated the NNT using the numbers that appeared in the first $\mathrm{RCT}^{1}{ }^{1}$ the NNT was 57 , suggesting amalgam was slightly more effective than glass ionomer cement. The result from another $\mathrm{RCT}^{2}$ was that the success rates for amalgam and glass ionomer were exactly the same, meaning the NNT did not converge. Taking account of results from other clinical studies, the variation within the research was too large. Even for the most investigated material, glass ionomer cement, it is hard to conclude whether it is superior or equivalent to amalgam. For the various other materials that have been introduced, clinical evidence is even more limited. Even MTA (mineral trioxide aggregate), widely accepted as a promising retrofilling material in the clinical setting in recent times, was supported only with case reports and in vitro studies. Further research is needed because no controlled trials have been performed.

Clinical superiority of retrofilling materials can be most effectively demonstrated by clinical studies. There is not enough evidence to recommend alternative retrofilling materials to amalgam at the present time.

\section{Practice point}

- At present the evidence is not strong enough to recommend a move from amalgam.

\section{Toru Naito}

Department of Periodontology and Endodontology, Kyushu Dental College, Kitakyushu, Japan

1. Zetterqvist L, Hall G, Holmlund A. Apicectomy: a comparative clinical study of amalgam and glass ionomer cement as apical sealants. Oral Surg Oral Med Oral Pathol 1991; 71:489-491.

2. Jesslen $P$, Zetterqvist $L$, Heimdahl A. Long-term results of amalgam versus glass ionomer cement as apical sealant after apicectomy. Oral Surg Oral Med Oral Pathol Oral Radiol Endod 1995; 79:101-103.

Evidence-Based Dentistry (2004) 5, 12.

doi: $10.1038 /$ sj.ebd. 6400235 